\title{
Virtual reality as therapeutic tool in the confines of social anxiety disorder treatment
}

Helena Grillon, BSc, MSc ${ }^{1}$, Françoise Riquier, BSc, MSc, PhD², Bruno Herbelin, BSc, MSc, PhD ${ }^{3}$, and Daniel Thalmann, BSc, MSc, $\mathrm{PhD}^{1}$

${ }^{1}$ VRLab, Swiss Federal Institute of Technology (EPFL), Lausanne, Switzerland; ${ }^{2}$ Psychiatrist and psychotherapist, Lausanne, Switzerland and ${ }^{3}$ Computer Science Department, Aalborg University Esbjerg, Denmark

\begin{abstract}
In this paper, we present a study, conducted over eight social phobic subjects, whose aim is to evaluate the efficiency and flexibility of virtual reality as a therapeutic tool in the confines of a social phobia behavioral therapeutic program. Our research protocol, accepted by the ethical commission of the cantonal hospices' psychiatry service, is identical in content and structure for each patient. This study's second goal is to use virtual exposure to evaluate objectively a specific parameter present in social phobia, namely eye contact avoidance, by using an eyetracking system. Analysis of our results shows a tendency to improvement in the subjects' feedback to specific assessment scales, which is correlated to the decrease of eye contact avoidance. The results show that the presented virtual reality exposure therapy protocol could be successfully applied to social therapy.
\end{abstract}

Keywords: Virtual reality exposure therapy, cognitive behavioral therapy, social phobia, fear of public speaking

Correspondence: Helena Grillon, BSc, MSc, VRLab, Swiss Federal Institute of Technology (EPFL), 1015 Lausanne, Switzerland. E-mail: helena.grillon@epfl.ch

Submitted: April 01, 2006. Revised: May 15, 2006. Accepted: May 16, 2006.

\section{INTRODUCTION}

The goal of our study is to define a therapeutic program for social anxiety disorders using virtual reality (VR) and to assess its efficiency to confirm that VR is a promising tool for psychotherapists in the confines of social phobia treatment. We equally introduce our eye-tracking device as a new tool for the assessment of social phobia and present the results we have obtained using it.

Nowadays, exposure to VR presents itself as an alternative to standard in vivo exposures in the context of cognitive and behavioral therapy (CBT). As of today, several studies have been conducted regarding the use of VR in the treatment of social phobia (1-4), all leading to the conclusion that VR immersion seems adequate for such treatments but all evaluated on limited sized cohorts. Anderson's study (5) equally evaluatesd the treatment on a small cohort (2 people). James (6) concluded that a socially demanding VR environment is more anxiety provoking for a phobic than for a non-socially demanding one. Slater (7) demonstrated that the difference in impact between an empty room and a room filled with avatars is more important in the case of phobics than in that of non-phobics. In her study, Klinger (8) concluded that both VRT (Virtual Reality Treatment) and CBT treatments are clinically valid and that the difference between the two is trivial.

Regarding eye contact, Horley (9) conducted a study on visual scanpath over 15 social phobic subjects and 15 nonphobic subjects. Her results suggest that the avoidance of salient facial features is an important marker of social phobia.
As a preliminary work, we conducted a study during which we exposed subjects to a VR situation representing a 3-dimensional audience composed of emergent gazes in the dark and surrounding the subject (10-11). We experimentally confirmed that the audience was able to provoke more anxiety to social phobics than to non-phobics and emitted the hypothesis that eye contact is an important factor of social phobia. We therefore developed and experimented with an eye-tracking setup integrated in the virtual reality exposure (VRE) system and concluded that eye-tracking technology could "provide therapists with an objective evaluation of gaze avoidance and can give tangible feedback to the patients to estimate their progress during gaze behavior exercises" $(12,62)$. The present paper is an extension of work first presented in ICDVRAT 2006 (13).

In the second section of this paper, we describe our research protocol; we then present our results in the third section before concluding.

\section{METHODS}

VR offers anxiety provoking scenarios that are not easily available in real life and are difficult to reproduce. As an example, for a therapist to fill his/her office with spiders in order to treat arachnophobia would be extremely difficult. Equally, to take a patient repeatedly on an airplane to treat him/her against fear of flights would be extremely expensive and time consuming. VR also allows repeating exposures without limitations. For example, a job interview is an accessible but exceptional situation. Having to do a job interview every week, as a habituation exercise, would be 
difficult. Our methodology is based on this idea and involves VR as a core component of the therapy.

\section{Selection and description of participants}

We recruited 10 subjects via a mailing sent to students in the second and third years of college and via ambulatory consultations specialized in anxiety disorders. The subjects were to be ages 18 to 45 years. We admitted the subjects to participate to the study after a structured interview regarding socio-demographical variables as well as psychiatric and medical antecedents. We led a diagnostic according to the DSM-IV's (19) 5 axis for each subject and presented them with the M.I.N.I. (Mini-International Neuropsychiatric Interview) (20) to verify the prevalence of social phobia and the absence of comorbidity. Exclusion criteria were the following:

- Previous or actual psychotic episode

- Addiction disorders

- A major depressive state or a scoring above 18 on Hamilton's scale (21)

- A significant somatic illness having an impact on social phobia or on the accomplishment of exposure

- A psychotherapy in progress

As two admitted participants dropped out during the A phase of the treatment, we conducted the study over eight subjects. They are referenced in this document by the letters A, E, G, I, C, K, O, and J, where A, E and $\mathrm{O}$ are males and $\mathrm{G}, \mathrm{I}, \mathrm{C}, \mathrm{K}$ and $\mathrm{J}$ are females.

\section{Technical information}

To evaluate the efficiency and the potential of VRE, we use one of the social situations that are most characteristic of social phobia according to Hofmann's model (14): the fear of public speaking. To this end, we have conceived a framework based that model by replacing the group exposure situations proposed in this therapy by individual exposure sessions to different virtual public speaking situations (Figures 1-4). Phobic subjects usually recourse to avoidance strategies concerning fearful situations. The aim of these exposures is to confront the subject to his/her fear and by habituation, make him/her cope with anxiety instead of avoiding it.

To evaluate the efficiency of our program, we use various scales specific to social anxiety disorders at different phases of the treatment, namely the Fear Questionnaire (15), the Liebowitz social anxiety questionnaire (16), the Social Interaction Self-Statement Test (17), and the Beck Depression Inventory (18). Our aim is to obtain a normalization of the score values for each subject after treatment as well as to uphold the improvement during the follow-up evaluations.

\section{Experimental methodology}

This project is a clinical experiment following an A-B protocol. During the A phase --the non-intervention phase--

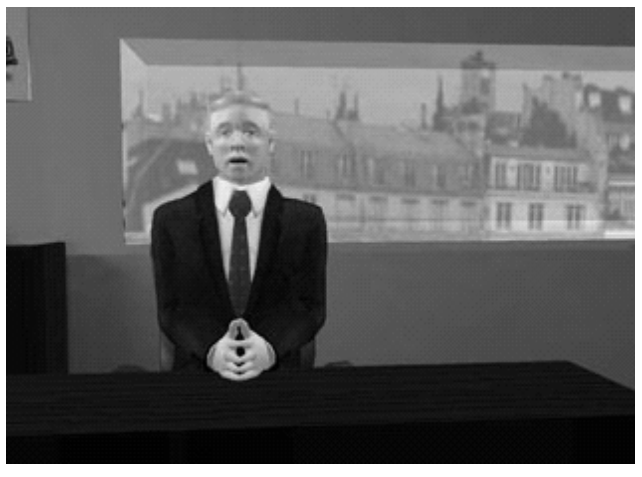

Fig. 1: Job interview simulation

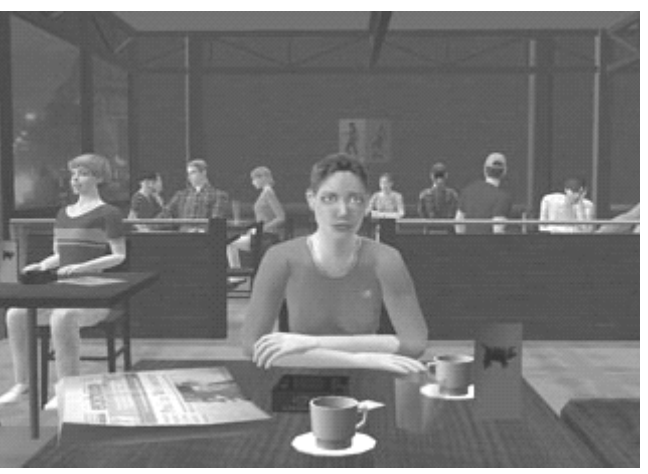

Fig. 2: Meeting in a bar

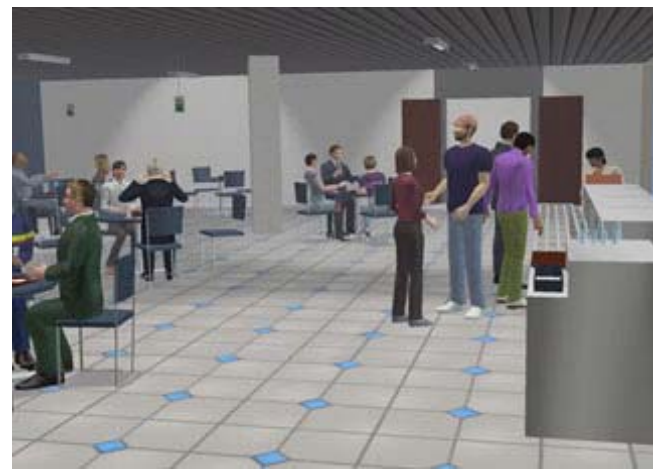

Fig. 3: Meeting in a cafeteria

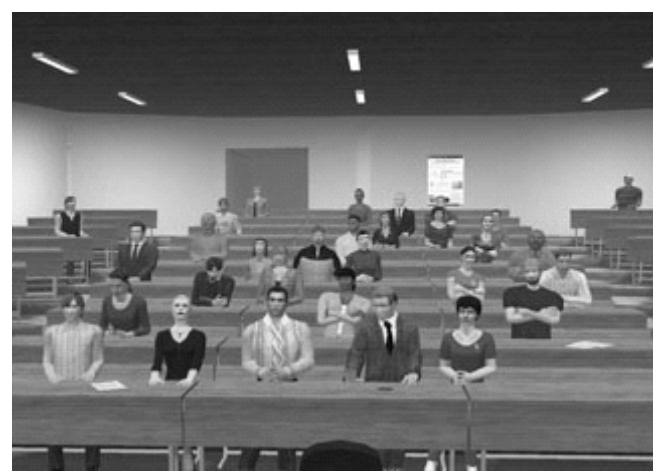

Fig. 4: Speech in an auditory 
we establish and analyze the target symptoms evolution curve through 3 evaluation sessions. During the B phasethe intervention phase-we expose the subjects to anxiety provoking situations through HMD immersion (V8 Head Mounted Display-Virtual Research Systems, Inc. 3824 Vienna Drive, Aptos, California 95003, USA) on a weekly basis, during 8 weeks; each session lasting $\sim 30$ minutes of which 10 in the HMD.

During the A phase, at weeks $-2,-1$, and 0 , we asked the subjects to fill in the above mentioned questionnaires (Fear Questionnaire, Liebowitz scale, SISST and BDI). We then analyzed them and averaged the results we obtained over the 3 weeks to obtain a before-treatment value for each subject and each scale.

Between phase A and phase B, the subjects participated in a group session without VR. We instructed them on social phobia and asked them, one after the other, to give a speech on what they had learned about this anxiety disorder.

For the B phase, we asked the subjects to mention 8 social situations and to classify them from least to most anxiety provoking. We then exposed them to various virtual situations throughout the 8 VR sessions, each more anxiety provoking than the previous one. The proposed virtual scenes were as follows:

- In an office, facing one man or one woman (Figure 1),

- In an office, facing five people,

- In an auditory, facing one man or one woman,

- In an auditory, facing approximately 20 people or sitting at the back of the room (Figure 4),

- In a cafeteria, facing one person but with many people around (Figure 3),

- In a bar, facing one person but with many people around (Figure 2).

It is known that dealing with a person of the opposite sex is a typical trait of social phobia. We therefore consider the office with one person (man or woman) and the auditory with one person (man or woman) as four different situations.

We have noticed that vocal interruptions from the therapist during HMD exposure created breaks in presence. We therefore avoided these by making our virtual characters talk instead. We set the virtual characters in each of these scenes with a number of pre-recorded sentences that can be triggered by the psychotherapist to respectively begin, continue, and end the speech session. We also provided the virtual characters with facial animation corresponding to the prerecorded sentences. Finally, we set up our characters with a "look at" function, which allows them to make eye contact at all time and more specifically when talking to the exposed subject.

For the first HMD session, we asked each subject to present social phobia once again, as they did for the group session. Then, each week, we asked them to prepare the following week's session. As homework, they had to prepare the following week's speech in front of a mirror in order to auto-evaluate their body language. Sessions 2 to 8 consisted in the following themes:

- Session 2: talk about hobbies,

- Session 3: talk about professional or educational activity,

- $\quad$ Session 4: talk about a memorable event,

- Session 5: talk about a dramatic situation,

- Session 6: talk about a conflict situation,

- Session 7: talk about anxiety related to love,

- Session 8: we gave them documents discussing "efficient communication" and asked them to talk about these documents as if giving a lecture.

We gave them these situations in this specific order for each to be more personal than the previous and therefore, more anxiety provoking. Because each subject is not affected by each situation in the same way, however, we modulated these according to each subject. As an example, some subjects recited a poem or sang a song for session 7 because talking about their love life wasn't sufficiently anxiety provoking.

As in Hofmann's therapeutic program, we asked, as homework, for the subjects to prepare and repeat speaking exercises in front of a mirror. We also asked them to try to decrease their avoidance behaviors in real life. Finally, we also asked them to fill in a "fearful situation" document in which they exposed the anxiety provoking situations to which they have been confronted in the past week as well as the degree of avoidance, the degree of anxiety, and the satisfaction felt throughout this experience. We then used this document as base to each weekly discussion. We asked the subjects to fill in the same 4 questionnaires as during the A phase of the treatment at week 5 (half way through the treatment) and at week 9 (after the end of the HMD sessions).

Regarding evaluation of visual contact avoidance, we use an eye-tracking system (ISCAN, Inc. 89 Cambridge Street, Burlington, MA 01803, USA), coupled with exposure to two virtual scenes with a 3-minute verbal expression exercise in each case. We evaluate eye contact avoidance from the recording of the pupil movements during exposure to the virtual scene. We seat the subject in front of a large back projection screen and equip him/her with the eye-tracking device. We then record the pupil movement at the rate of 60 measures per second. Finally, we analyze this recording and materialize the data as a map showing the zones swept by the gaze as well as the lapse of time contact lasted. Our eyetracking system allows us to put into evidence and analyze the virtual environment's zones, which are looked at by the subject in real time (Figure 5).

We conducted the eye-tracking tests before and after the HMD sessions to analyze the progression in eye contact before and after treatment. Before starting with the first session, we exposed the subjects to a 5 minute on the screen with their eyes). This was done to habituate them to the equipment and to relax before exposure by playing a game. 


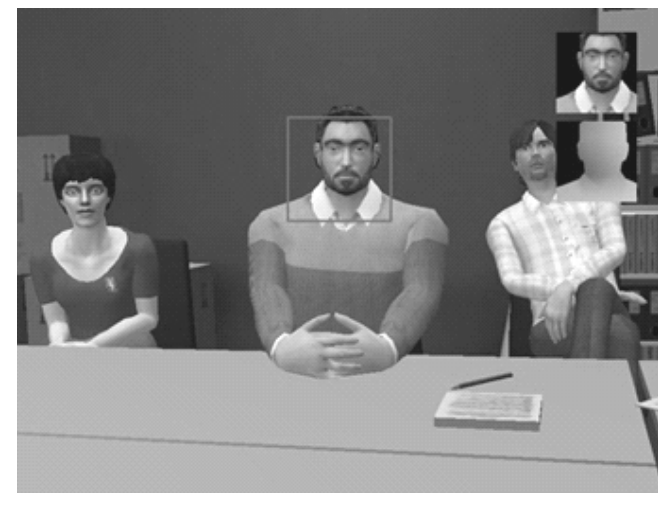

Fig. 5: Eye-tracking system visual demonstration

During both sessions, we set the subjects in front of two different scenes, facing one man sitting in his office (figure 1) and facing an auditory containing an audience of approximately 20 virtual actors (figure 4). We did these two recordings in order to check whether the eye contact attitude is the same in different situations or not.

Once the second eye-tracking session was terminated, we conducted a final group session. During this group session, we asked the subjects to present their lecture on efficient communication once again, in front of the others. Finally, our protocol plans a follow-up session, 24 weeks after the end of the treatment to verify the presence of continued evolution.

\section{RESULTS}

First of all, we note a general improvement for most subjects through the analysis of the various questionnaires.
We can equally see that the tendencies for each subject are repeated throughout all questionnaires. We also note that visual contact avoidance decreases. Our results show that the subjects present less avoidance behavior after treatment than before treatment. We equally note that one person out of the eight does not follow the improvement pattern, on the contrary. We can see that the evolution of this subject is the opposite to that of all other subjects (subject $G$ ). If we took out this subject from our study, we would have a much better mean evolution.

\section{Questionnaire analysis}

In the mean, we can see that the social phobia score for the Fear Questionnaire has decreased from 22.3 to 16.4 for women and from 14.7 to 11.3 for men. The norms to this questionnaire for phobic subjects are of respectively 15.94(8.96) to 23.4(8.4) for women and 21.4(5.44) to 24.4(8.0) for men (22). Our results are therefore promising.

Regarding the Liebowitz questionnaire, the mean score decreased from 68.6 to 50.9. Knowing that 67.2(27.5) is the norm for social phobic subjects (22), our results are equally promising. In the mean, the score to the SISST positive thoughts has evolved from 39.0 to 46.1 and the score to the SISST negative thoughts, decreased from 45.3 to 36.1 . For phobic subjects, the norms to this test are of 36.93(7.40) and 53.46(9.11) for positive and negative thoughts respectively (22). Once again, the results confirm those of previous tests. For the BDI questionnaire, the mean score has decreased from 8.6 to 4.8. Knowing that a score of 18.7(10.2) denotes a slight depression and that a score of $10.9(8.1)$ denotes no depression whatsoever (22), we can conclude that our subjects were not depressive, or very slightly depressive,

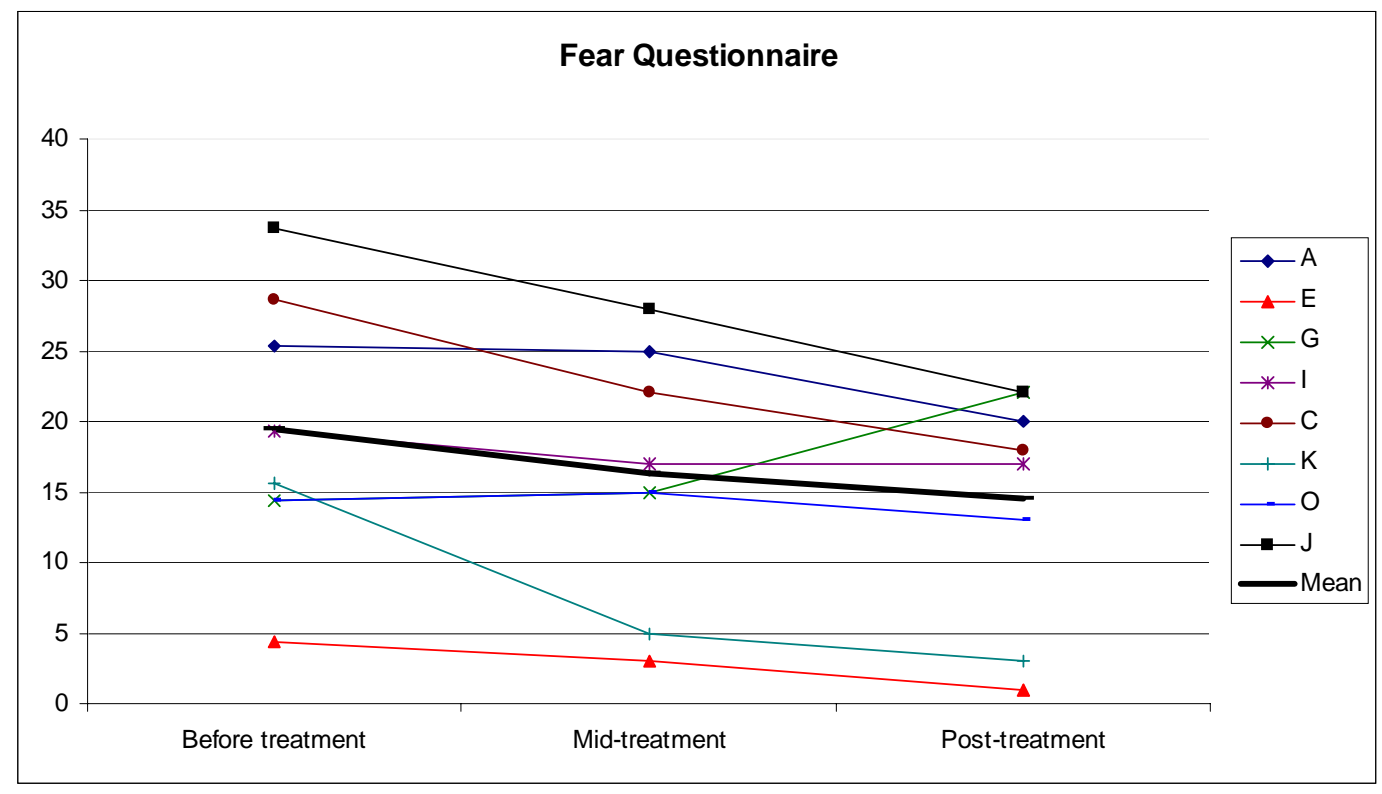

Fig. 6: Graphical results to the Fear Questionnaire 


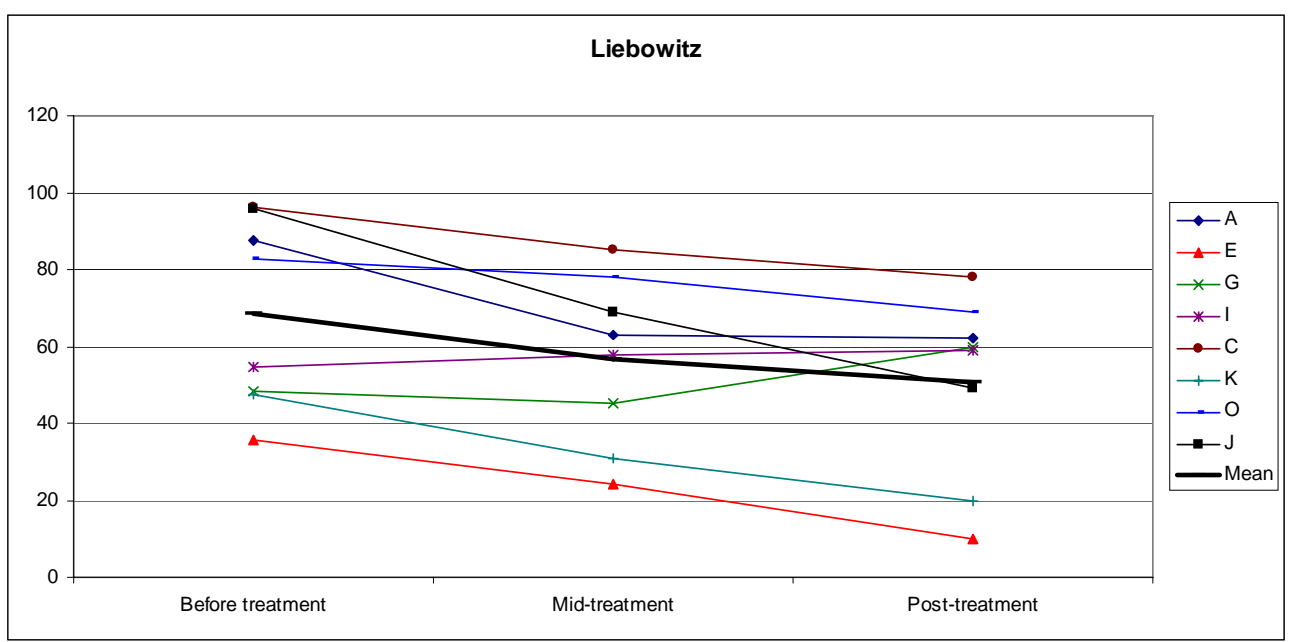

Fig. 7: Graphical results to the Liebowitz Questionnaire

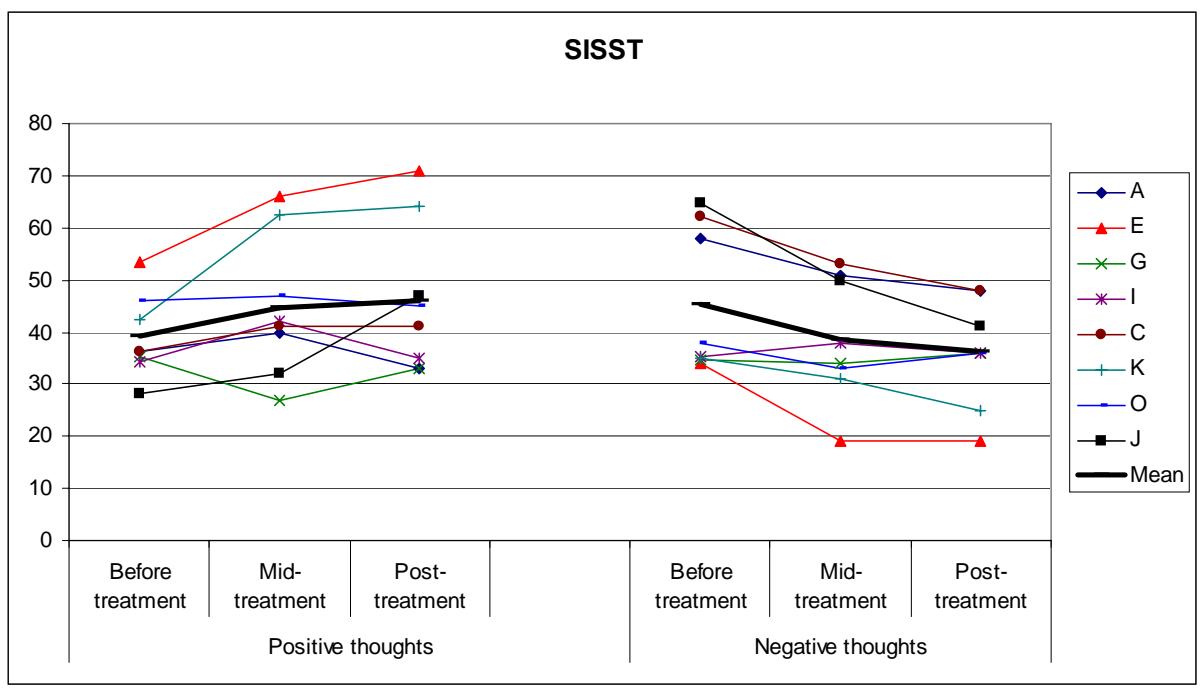

Fig. 8: Graphical results to the SISST Questionnaire

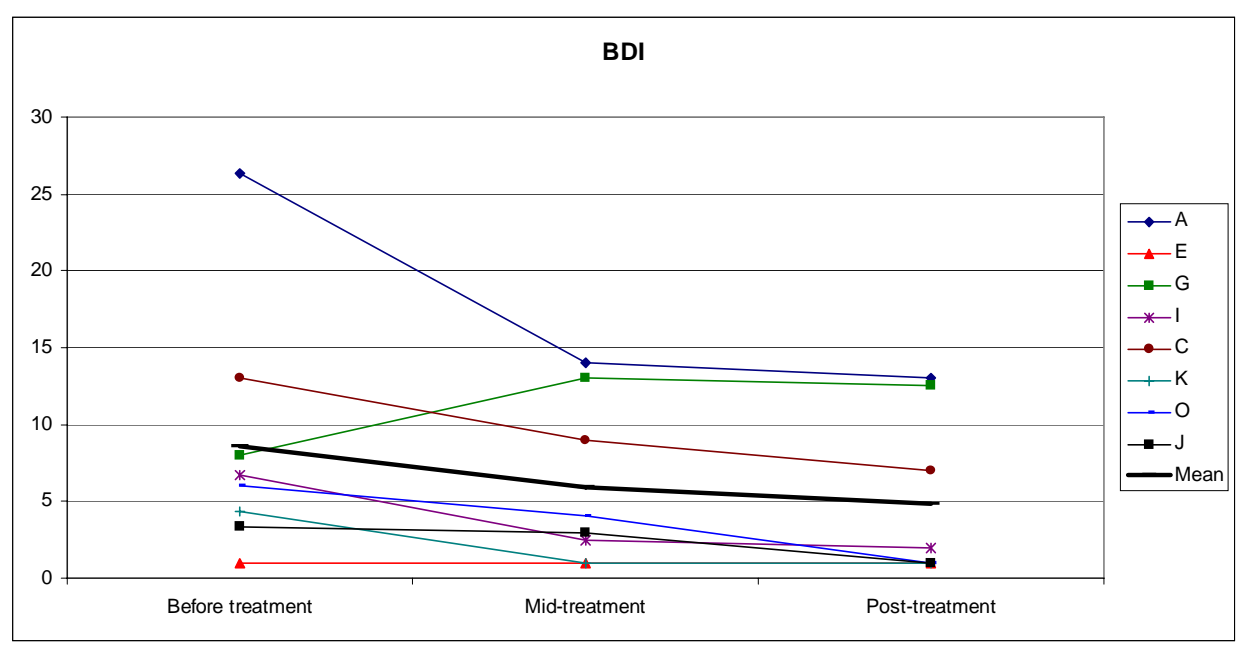

Fig. 9: Graphical results to the BDI Questionnaire 
should it be at the beginning of the treatment or after its end. We can also see that subject " $A$ " had a much higher score than all other subjects. From our results, this subject had a moderate depression at the beginning of the treatment and mild depression at the end of the treatment. All other subjects had either no depression or mild depression at the beginning of treatment.

\section{Eye-tracking}

The evolution in eye contact is different in each case; we have noticed, however, a general improvement in eye contact avoidance when comparing the results of the eye- tracking sessions before and after treatment. Of the eight subjects, four showed noticeable improvement in the first scene and six in the second scene (Figures 10,11). One subject showed no improvement between the two sessions and for both scenes (Figure 12). This is subject " $G$ ”, the same one that had results contrary to all others regarding scores to the questionnaires. For the first scene, two subjects already had good results in the pre-treatment session and therefore did not show any improvement (Figure 13). Finally, we noticed that we had problems in the calibration process for one subject for the pre-treatment session. This subject's results, therefore, cannot be analyzed.
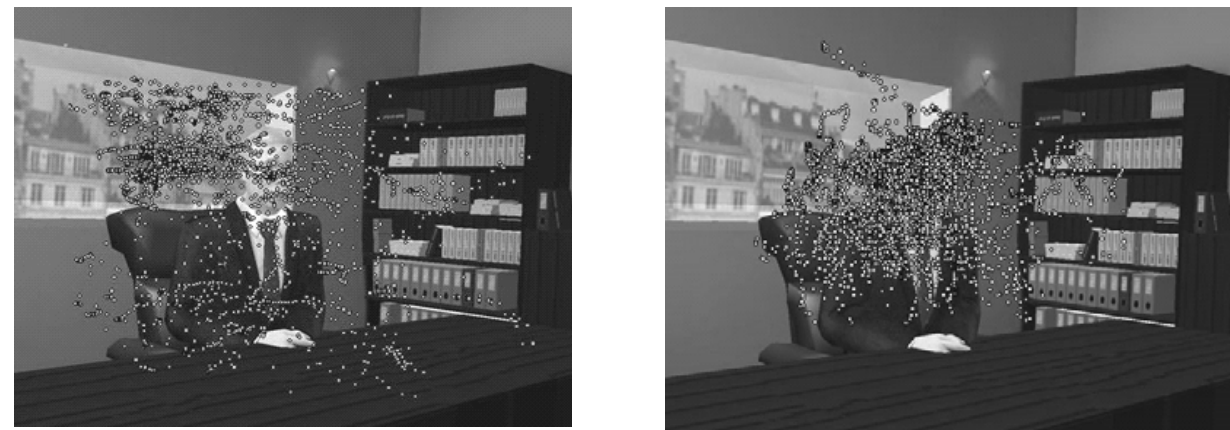

Fig. 10: Eye-tracking results for the interview simulation - improvement case. Left: before treatment Right: after treatment.
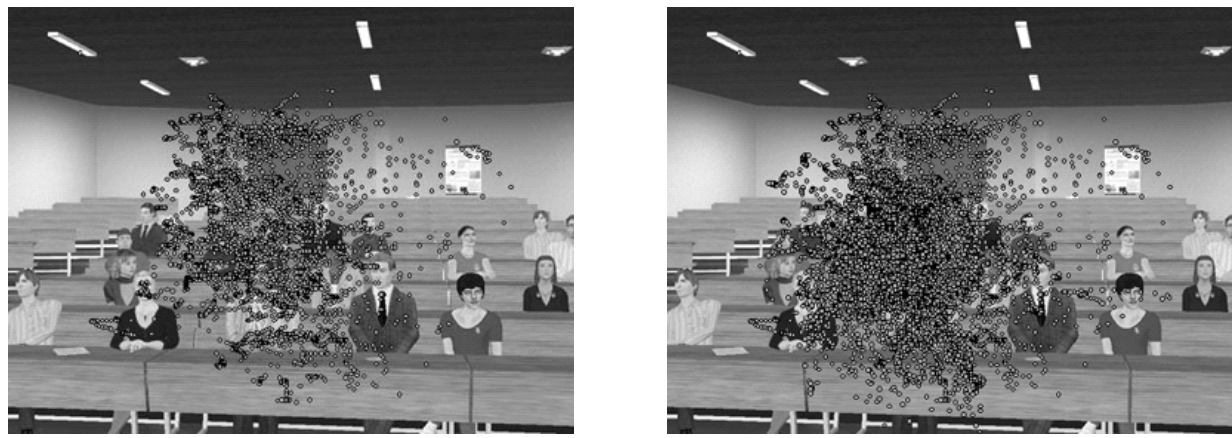

Fig. 11: Eye-tracking results for the speech simulation - improvement case. Left: before treatment Right: after treatment
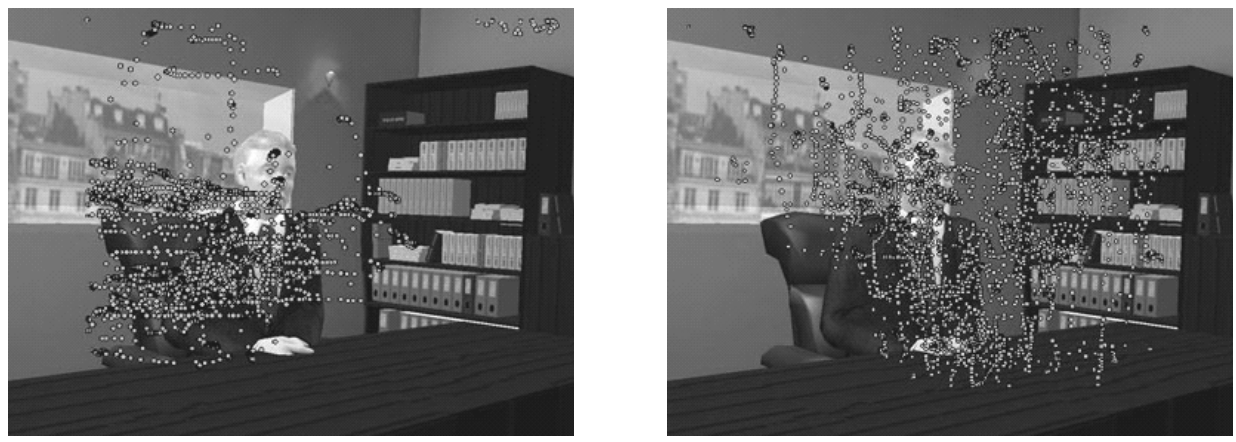

Fig. 12: Eye-tracking results for the interview simulation - no improvement case. Left: before treatment Right: after treatment 

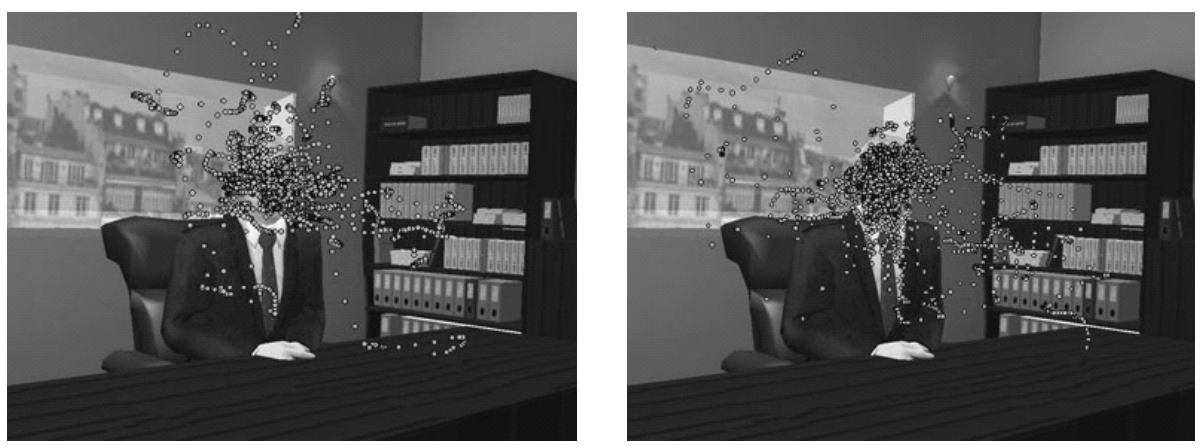

Fig. 13: Eye-tracking results for the interview simulation - both good case. Left: before treatment, Right: after treatment

From these recordings, it is noticeable that after treatment, eye contact behavior changed for most subjects. For subjects who have shown noticeable improvement (4 out of 7 in the first scene and 6 out of 7 in the second scene), we can notice that the virtual character's face is looked at much more after the end of the treatment than before treatment (Figure 10). We can see that the talking virtual characters (the lady in red and the man with the white sweatshirt, respectively) are equally much more looked at after treatment than before treatment in the second situation (Figure 11). The results tend to show that eye-contact avoidance has diminished after treatment in most cases and that looking at the talking person's face has become less difficult.

In the case of subject " $G$ ", eye contact behavior has changed between pre-treatment and post-treatment phases. However, these changes show no improvement (figure 12). The analysis of eye tracking data in such a case requires more investigations from the psychological point of view. It allows the highlight of changes in the avoidance strategy, which denotes a shift in the patient's reassurance behaviors. Correlations between centers of interest and the subject's verbal responses offer a possible lead for the therapist. That certain subjects demonstrated good results in the pretreatment phase as well as in the post-treatment phase (Figure 13) shows that not all social phobic subjects react in the same way and suggests that there are different types of social phobias.

\section{DISCUSSION}

In this paper, we have presented a study whose aim was to evaluate the efficiency of a therapeutic program using VR as a tool to treat social phobia. We have presented the VRbased protocol that we have used to conduct a study over eight social phobic subjects. We have equally presented the results we obtained through questionnaire analysis and eyetracking recordings.

We equally noticed that the tendencies for each subject were the same over the questionnaires. Moreover, the same tendencies are equally visible for the eye-tracking. First, we noticed a general improvement for most subjects after having analyzed all questionnaires. We have seen, in our results, that one of the subjects apparently was not affected by the treatment at all, which leads us to think that certain people are more reactive to VRET than others.

Due to the limited size of our sample, we cannot conclude about the validation of VR as a treatment for social phobia. Nevertheless, our results show that VR is a valuable therapeutic tool and that it offers different perspectives to be investigated. Another study should be conducted over a minimum of 30 subjects and with a control group to validate our protocol fully. Fifty subjects should be recruited, however, because some of them will certainly drop out before the end of the study, as we have seen in ours.

Secondly, and according to our former experience in case studies (12), we observed a deviation of the gaze during VRE and obtained a qualitative appreciation of the patients' gaze avoidance. After having analyzed the eyetracking recordings, we equally noticed an improvement in eye-contact avoidance. We are currently working on qualitative evaluation tools for this behavior.

By using subjective appreciation of social anxiety throughout the whole experiment (the four questionnaires mentioned above), as well as video recordings of the exposure sessions and the results provided by our eyetracking system, we noticed an improvement regarding avoidance behavior and a decrease of anxiety with time and exposure. The follow-up with the subjects will give us the opportunity to validate our actual results regarding VR therapy on a long-term basis and its ability to continue providing results once the therapy is over.

\section{ACKNOWLEDGMENTS}

We would like to thank Diana Felli and Caroline Nicod for having helped us conduct the HMD sessions, Mireille Clavien for the design and Benoît Le Callennec for careful proofreading. Part of the software has been developed thanks to a Swiss National Research Foundation grant.

\section{REFERENCES}

1. North M, North S and Coble JR. Virtual reality therapy: an effective treatment for the fear of public speaking. IJVR 1998;3(2):2-6.

2. Pertaub D, Slater M and Barker C. An Experiment on fear of public speaking in virtual reality. Stud Health Technol Inform 2001;81:372-8. 
3. Pertaub D, Slater M and Barker C. An Experiment on fear of public speaking anxiety in response to three different types of virtual audience. Presence 2002;11(1):68-78.

4. Harris SR, Kemmerling RL and North MM. Brief virtual reality therapy for public speaking anxiety. CyberPsychol Behav 2002;5(6):543-50.

5. Anderson P, Rothbaum BO and Hodges LF. Virtual reality in the treatment of social anxiety: Two case reports. Cogn Behav Pract 2003;10:240-7.

6. James LK, Lin CY, Steed A, Swapp D and Slater M. Social anxiety in virtual environments: results of a pilot study. CyberPsychol Behav 2003;6(3):237-43.

7. Slater M, Pertaub DP, Barker C and Clark D. An experimental Study on fear of public speaking using a virtual environment. IWVR, Lausanne, 16-17 September 2004.

8. Klinger E, Bouchard S, Légeron P, Roy S, Lauer F, Chemin I and Nugues P. Virtual reality therapy versus cognitive behavior therapy for social phobia : A preliminary controlled study. CyberPsychol Behav 2005;8(1):76-88.

9. Horley K, Williams LM, Gonsalvez C and Gordon E. Social phobics do not see eye to eye: A visual scanpath study of emotional expression processing. J Anxiety Disord 2001;17:33-44.

10. Herbelin B, Riquier F, Vexo F and Thalmann D. Virtual reality in cognitive behavioral therapy: a preliminary study on social anxiety disorder. VSMM, Gyeongju, 25-27 September 2002

11. Riquier F, Stankovic M and Chevalley AF. Virtual gazes for social exposure: Margot and Snow White. VRMHR, Lausanne, 7-8 November 2002, 35-49.

12. Herbelin B. Virtual reality exposure therapy for social phobia. PhD Thesis $\mathrm{N}^{\circ}$ 3351, VRLab, Faculté informatique et communications, Institut des systèmes informatiques et mutlimédias, EPFL, Switzerland, 2005.

13. Grillon H, Riquier F, Herbelin B and Thalmann D.
Use of virtual reality as therapeutic tool for behavioral exposure in the ambit of social anxiety diorder treatment. ICDVRAT, Esbjerg, 18-20 September 2006, 105-112.

14. Hofmann SG. Self-focused exposure therapy for social phobia. Unpublished treatment manual, Boston Univ, 1997.

15. Marks IM and Matthews AM. Brief standard selfrating for phobic patients. Behav Res Ther 1979;17: 263-7.

16. Yao SN, Note I, Fanget F, Albuisson E, Bouvard M, Jalenques I and Cottraux J. L'anxiété sociale chez les phobiques sociaux : validation de l'échelle d'anxiété sociale de Liebowitz. Encephale 1999;25:429-35

17. Yao SN, Cottraux J, Mollard E, Albuisson E, Note I, Jalenques I, Fanget F, Robbe-Grillet P, Dechassey M, Ektmedjian N, Bouvard M and Coudert AJ. The french version of the social interaction self-statement test (SISST): a validation and sensitivity study in social phobics. Behav Cogn Psychother 1998;26:247-59.

18. Beck AT, Ward $\mathrm{CH}$, Mendelson M, Mock J and Erbaugh J. An inventory for measuring depression. Arch Gen Psychiatry 1961;4:561-71.

19. Diagnostic and Statistical Manual of Mental Disorders (DSM-IV). Washington, DC: Am Psychiatr Assoc, 1994.

20. Sheehan DV, Lecrubier $Y$, Sheehan KH, Amorim P, Janavs J, Weiller E, Hergueta T, Baker R and Dunbar GC. The Mini-International Neuropsychiatric Interview (M.I.N.I.): the development and validation of a structured diagnostic psychiatric interview for DSM-IV and ICD-10. J Clin Psychiatry 1998;59:22-33.

21. Hamilton M. Development of a rating scale for primary depressive illness. $\mathrm{Br} \mathrm{J}$ Soc Clin Psychol 1967;6:278-96.

22. Bouvard $M$ and Cottraux J. Protocoles et échelles d'évaluation en psychiatrie et en psychologie. Paris: Masson, 2002. [French] 